\title{
On Mathematical Model of Biological Pest Control of Sorghum Production
}

\author{
Y. O. Aderinto*, I. T. Issa, A. O. Afolabi and O. M. Bamigbola \\ Department of Mathematics, Faculty of Physical Sciences, University of Ilorin, P.M.B. 1515, Ilorin, \\ Nigeria \\ e-mail: aderinto@unilorin.edu.ng; Temitopeissa@gmail.com; afolabiahmed79@gmail.com; \\ ombamigbola@gmail.com
}

\begin{abstract}
In this paper, mathematical model of Sorghum production from seed/planting to harvesting stage was presented with respect to its pests(prey) and the corresponding natural prey enemy (predators) at every stage. The model was characterized, the existence and uniqueness of the model solution was established. And finally numerical applications was carried out using Differential Transform method, and it was found that pests at different stages of sorghum production can be minimized below injury level using biological control which in turn leads to maximization of the sorghum production.
\end{abstract}

\section{Introduction}

An important cereal crop known as Sorghum (Sorghum bicolor L. Moench) is widely grown in arid and semi-arid ecology worldwide for food and feed purposes. It can be reffers to as a security crop because it has a wide adaptability and tolerant to environmental stress. The production of sorghum is drastically affected by different kind of diseases and pests such as Grasshopper, Weevil, Sugarcane beetle, Leaffooted bug, Mites, Wire worm, Aphids and Army worm, etc. In another development litterature revealed that there are some beneficial group of arthropods, such as lady beetle (ladybug or lady bird) which are natural enemies of many sorghum insectpests especially aphids, mites and others. Single lady beetle may eat as many as 5,000 aphids in its lifetime, [10]. Lady bugs has different species and most species are considered beneficial pest because

Received: December 3, 2021; Accepted: January 9, 2022

2020 Mathematics Subject Classification: 49-XX, 49J15, 49K15, 49Mxx.

Keywords and phrases: biological control, sorghum production, pest, prey and predator, existence and uniqueness. 
they are predators of many harmful pest. This contribute to the high level of regulations of prey population, though ladybird larvae and adults may supplement their normal prey in times of scarcity with other types of food like flower nectar, water and honeydew on sorghum panicle, [6].

Sorghum as a member of the grass family is classified into Grain Sorghum, Sweet Sorghum, Broom Sorghum, and Grass Sorghum. It was ranked second among cereals and fifth among all crops in terms of population in Africa. Its production represents $25 \%$ of total cereal production in Nigeria in the year 2012. It is recognized by United State Department of Agriculture as a primary food crop produced in virtually all parts of Northern Nigeria, however demand from industries is the main drive behind increase in sorghum production. Though Boko Haram insurgencies continue to limit land for sorghum production in the northeastern part of Nigeria but farmers have continued to be encouraged by increasing prices and rising sorghum demand, both for food and for industrial use over the last two years, [7, 8, 14, 15].

Sorghum has multiple uses as grain forage, fodder, and more recently as bioenergy crop. In Asia and Africa, sorghum grain is consumed by humans or as animal feed, its stalks are used as animal fodder, or as housing material. It has a high nutritive value, with $70-80 \%$ carbohydrate, $11-13 \%$ protein, $2.5 \%$ fat, $1-3 \%$ fiber and $1-2 \%$ ash. Protein in sorghum is gluten free and thus, it is a specialty food for people who suffer from celiac (intolerance to food with gluten), including diabetic patients, [17].

Many researchers have worked on sorghum analysis, Sisterson and Stenger [12] carried out a research with the aim of developing a prediction equation describing the cleaning process in a stationary sorghum thresher using a mathematical model based on physical-aerodynamic properties of sorghum and machine characteristics. [3] studied the effect of high temperature on crop growth development, in vitro pollen germination and seed set. [11] studied new sorghum pest in North America (sugarcane aphid). [13] studied the mathematical characterization of growth of a local land-race sorghum from Saudi Arabia (Jazan Region).

In another development, many authors have used the concept of mathematics to model and analyse dynamics of real life situations. Among are: Egbetade and Ibrahim [5], researched on a mathematical model of tuberculosis (TB) incorporating treatment, migration and vaccination, with the focus on vaccination for the treatment of active TB. [9] developed a model to analysed the strategies to control pest through differential equations. [1] investigates the application of differential transformation method (DTM) 
and variational iteration method (VIM) in finding the approximate solution of epidemiology (SIR) model. [4] propose a mathematical model on the stability analysis of a non-linear HIV / AIDS epidemic with vaccination and antiretroviral therapy to mention a few. However, little or less has been studied on the mathematical dynamics of Sorghum production, hence the present effort is to look at the mathematical model of biological control of Sorghum pest from planting to harvesting stages.

\section{Model Formulation}

A deterministic dynamical model was formulated into two categories of population, namely: seeds/plant and insect population. The seed/plant population is further classified into seed $S c(t)$, germination $G(t)$, crop $C(t)$ and harvest $(H)$, while the insect population is categorized into two: the beneficial insect population $P_{v}(t)$ which could there after become harmful to sorghum seeds and pest population $I_{v}(t)$ which they are right from time harmful to sorghum. The model shows the movement from one growth stage to another at different rate. $\Lambda_{c}$ is the quantity of replenishing seed for plantation, $\frac{\beta_{1} I_{v}}{N+b I_{v}}$ is the force of infection of seed by pest. $\gamma$ and $\mu$ are the removal rate from germination class where $\gamma$ is the proportion that produces crop and $\mu$ denote natural bad seeds. $C$ is the quantity of matured crop that farmers will be able to harvest.

\section{Basic assumption of the model}

In constructing the model the following assumptions were used;

(i) The growth and development stages of sorghum were formulated in the model from planting of seed to the harvesting stage.

(ii) The stages of sorghum production examined are in homogeneous form, vectors can attack every stages of sorghum.

(iii) The Model is considered as a prey-predator population.

(iv) In the assurance of no pest, there exists no germination.

(v) The seeds are systemic.

(vi) Some pests have negative economic importance on sorghum while some have both positive and negative economic importance. 
The parameters of the model considered are defined in Table 2.1.

Table 2.1: Description of parameters and variables. The values are hypothetical.

\begin{tabular}{|c|c|c|}
\hline $\begin{array}{l}\text { Parameter/ } \\
\text { variables }\end{array}$ & Description & Value \\
\hline$\Lambda_{c}$ & Amount of seed replenishing & 50 \\
\hline$\beta_{1}$ & Probability of successful harm on sorghum seed. & {$[0,1]$} \\
\hline $\mathrm{N}$ & Total number of seed & 80 \\
\hline$\gamma$ & Percentage of germinating seed producing crop. & 0.6 \\
\hline$\epsilon$ & Fraction of crop which successfully harvest. & 0.7 \\
\hline$\mu$ & Amount of natural bad seed/natural loss on seeds. & 0.1 \\
\hline$\Lambda_{p}$ & $\begin{array}{l}\text { Relative recruitment rate for insect with both negative and } \\
\text { positive impact. }\end{array}$ & 15 \\
\hline$\Lambda_{v}$ & Relative recruitment rate for insect with negative impact. & 50 \\
\hline$b I_{v}$ & Proportion of pest interacting/having contact with seeds. & 5.1421 \\
\hline$\mu_{1}$ & Natural death rate for insects. & 0.1 \\
\hline$\alpha$ & Progressive of destructive insects. & 0.02 \\
\hline$S_{c}$ & Sorghum Seeds & 50 \\
\hline$G$ & Germination Stage & 35 \\
\hline C & Cropping & 30 \\
\hline$H$ & Harvest & 20 \\
\hline$P_{v}$ & Beneficial Pest & 7.5 \\
\hline$I_{v}$ & Pest & 10.285 \\
\hline
\end{tabular}

\subsection{The simple seed-germination-cropping-harvest (SGCH) model flow diagram}

In view of the assumptions we have the systematic diagram of the model. 


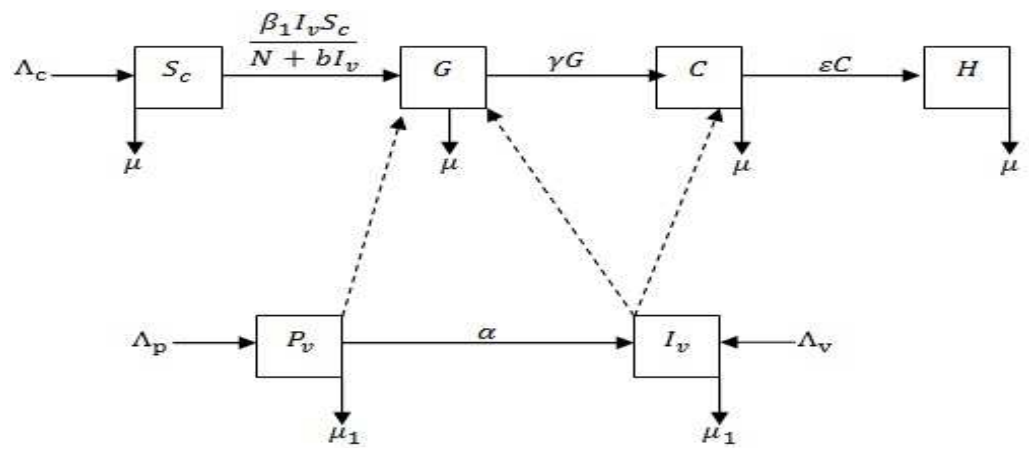

Figure 2.1: Systematic diagram of the sorghum production.

\subsection{Model Equation}

In view of the aforementioned assumptions, Figure 2.1 is obtained by the following first order nonlinear system of equations;

$$
\left.\begin{array}{rl}
\frac{d S_{c}}{d t} & =\Lambda_{c}-\mu S_{c}-\frac{\beta_{1} I_{v} S_{c}}{N+b I_{v}} \\
\frac{d G}{d t} & =\frac{\beta_{1} I_{v} S_{c}}{N+b I_{v}}-(\gamma+\mu) G \\
\frac{d C}{d t} & =\gamma G-(\epsilon+\mu) C \\
\frac{d H}{d t} & =\epsilon C-\mu H \\
\frac{d P_{v}}{d t} & =\Lambda_{p}-\mu_{1} P_{v}-\alpha P_{v} \\
\frac{d I_{v}}{d t} & =\Lambda_{v}+\alpha P_{v}-\mu_{1} I_{v}
\end{array}\right\}
$$

\section{Characterization of the Model}

The intention is to investigate the behavior of the model formulated.

\subsection{Region of feasibility and positivity of solution}

Theorem 3.1. Let $\Gamma$ be the feasible region of sorghum model system (1) defined as $\Gamma_{1}=\left\{(S c, G, C, H) \in R_{+}^{4}: S c+G+C+H \leq \frac{\Lambda}{\mu}\right\}, \Gamma_{2}=\left\{\left(P_{v}, I_{v}\right) \in R_{+}^{2}: N_{v} \leq \frac{\Lambda_{v}}{\mu_{1}}\right\} \quad$ and $\forall S C>0, G>0, C>0, H>0, \Gamma=\left\{\Gamma_{1}, \Gamma_{2}\right\} \in R_{+}^{6}$. Then $\Gamma$ is the positive invariant set.

Proof. Assume that $N=S_{c}+C+H$ and $G=0 I_{v}=0 . G=0$ (because when the seed started to germinate it turns to a plant and it is no more a seed as we are referring to a seed, until it turn to a crop, that is when you can get a seed again). 
Let

$$
\begin{aligned}
& N=S_{c}+C+H \\
\frac{d N}{d t}= & \Lambda_{c}-\mu S_{c}-\mu C-\mu H \\
= & \Lambda_{c}-\mu N
\end{aligned}
$$

At equilibrium

$$
\begin{gathered}
\Lambda_{c}-\mu N=0 \\
N(t)=\frac{\Lambda_{c}}{\mu}+C e^{-\mu t} \\
\left.N(t)\right|_{0} ^{\infty}=\frac{\Lambda_{c}}{\mu}+\left.C e^{-\mu t}\right|_{0} ^{\infty} \\
=\frac{\Lambda_{c}}{\mu}+C \\
C=\lim _{t \rightarrow \infty}\left(N(\infty)-N(0)-\frac{\Lambda_{c}}{\mu}\right)=N_{0}-\frac{\Lambda_{c}}{\mu}
\end{gathered}
$$

Put (4) in (3), we have

$$
=\frac{\Lambda_{c}}{\mu}\left(1-e^{-\mu t}\right)+N_{0} e^{-\mu t}>0, \quad \forall t
$$

Similarly, for pest population;

Let

$$
N_{v}=P_{v}+I_{v}
$$

Assume

$$
\begin{gathered}
\Lambda_{p}+\Lambda_{v}=\Lambda, N_{v}=\Lambda_{p}+\Lambda_{v}-\mu_{1} N_{v}, \\
\frac{d N_{v}}{d t}=\Lambda-\mu_{1} N_{v} \\
N_{v}(t)=\frac{\Lambda}{\mu_{1}}+C e^{-\mu_{1} t}
\end{gathered}
$$

At $t=0$

$$
\begin{gathered}
N_{v}(0)=\frac{\Lambda}{\mu_{1}}+C \\
N_{v}(t)=\frac{\Lambda}{\mu_{1}}+\left(N_{v}(0)-\frac{\Lambda}{\mu_{v}}\right) e^{-\mu_{1} t}
\end{gathered}
$$




$$
=\frac{\Lambda}{\mu_{1}}\left(1-e^{-\mu_{1} t}\right)+N_{v}(0) e^{-\mu_{1} t}>0, \quad \forall t
$$

By (4a) and (5a), hence the result.

\subsection{Existence and uniqueness of solution to the model}

In this subsection the existence and uniqueness of solution to model (1) shall be invesitigated using Lipschitz condition and continuous differentiability.

Lemma 3.1 [16]. Let $D \subset \mathbb{R}^{n}$ and $f: D \rightarrow \mathbb{R}$ be a nonlinear vector field. $f$ is continuous and Lipschitz in $B=\left\{x \in D:\left\|x-x_{0}\right\| \leq r\right\}$ for some real $r$ with $r>0$. Then, there exists some $\delta>0$ such that $x^{\prime}=f(t, x), x\left(t_{0}\right)=x_{0}$, has unique solution in $[0, \delta]$.

Theorem 3.2. Suppose $F_{i}(t, x), x\left(t_{0}\right)=x_{0}, i=1, \ldots, 6$ exists and unique in solution. Then the system equation (1) satisfies Lipschitz condition.

Proof. It is sufficient to show that $\frac{\partial f_{i}}{\partial t_{j}}, i, j=1,2,3,4,5,6$ are continuous and bounded in $D$.

Let

$$
\left.\begin{array}{l}
f_{1}=\Lambda_{c}-\mu S_{c}-\frac{\beta_{1} I_{v} S_{c}}{N+b I_{v}} \\
f_{2}=\frac{\beta_{1} I_{v} S_{c}}{N+b I_{v}}-(\gamma+\mu) G \\
f_{3}=\gamma G-(\epsilon+\mu) C \\
f_{4}=\epsilon C-\mu H \\
f_{5}=\Lambda_{p}-\mu_{1} P_{v}-\alpha P_{v} \\
f_{6}=\Lambda_{v}+\alpha P_{v}-\mu_{1} I_{v}
\end{array}\right\}
$$

Taking the partial derivatives of $f_{1}, f_{2}, f_{3}, f_{4}, f_{5}, f_{6}$ in equation (6) to obtain the following:

For $f_{1}$,

$$
\left.\begin{array}{l}
\left|\frac{\partial f_{1}}{\partial S_{c}}\right|=\left|-\mu-\frac{\beta_{1} I_{v}}{N+b I_{v}}\right|<\infty \\
\left|\frac{\partial f_{1}}{\partial G}\right|=\left|\frac{\partial f_{1}}{\partial C}\right|=\left|\frac{\partial f_{1}}{\partial H}\right|=\left|\frac{\partial f_{1}}{\partial P_{v}}\right|=0<\infty \\
\left|\frac{\partial f_{1}}{\partial I_{v}}\right|=\left|-\frac{N \beta_{1} S_{C}}{\left(N+b I_{v}\right)^{2}}\right|<\infty ; \quad \forall N+b I_{v} \neq 0
\end{array}\right\}
$$

For $f_{2}$ in (6), we have: 


$$
\left.\begin{array}{rl}
\left|\frac{\partial f_{2}}{\partial S_{c}}\right| & =\left|\frac{\beta_{1} I_{v}}{N+b I_{v}}\right|<\infty \\
\left|\frac{\partial f_{2}}{\partial G}\right| & =|-(\gamma+\mu)|<\infty \\
\left|\frac{\partial f_{2}}{\partial C}\right| & =\left|\frac{\partial f_{2}}{\partial H}\right|=\left|\frac{\partial f_{2}}{\partial P_{v}}\right|=0<\infty \\
\left|\frac{\partial f_{2}}{\partial I_{v}}\right| & =\left|-\frac{\beta_{1} S_{c}}{\left(N+b I_{v}\right)^{2}}\right|<\infty ; \quad \forall N+b I_{v} \neq 0
\end{array}\right\}
$$

Also for $f_{3}$,

$$
\left.\begin{array}{l}
\left|\frac{\partial f_{3}}{\partial S_{c}}\right|=0<\infty \\
\left|\frac{\partial f_{3}}{\partial G}\right|=|\gamma|<\infty \\
\left|\frac{\partial f_{3}}{\partial C}\right|=|-(\mu+\epsilon)|<\infty \\
\left|\frac{\partial f_{3}}{\partial H}\right|=\left|\frac{\partial f_{3}}{\partial P_{v}}\right|=\left|\frac{\partial f_{3}}{\partial I_{v}}\right|=0<\infty
\end{array}\right\}
$$

For $f_{4}$, we have:

$$
\left.\begin{array}{l}
\left|\frac{\partial f_{4}}{\partial S_{c}}\right|=0<\infty \\
\left|\frac{\partial f_{4}}{\partial G}\right|=0<\infty \\
\left|\frac{\partial f_{4}}{\partial C}\right|=|\epsilon|<\infty \\
\left|\frac{\partial f_{4}}{\partial H}\right|=|-\mu|<\infty \\
\left|\frac{\partial f_{4}}{\partial P_{v}}\right|=\left|\frac{\partial f_{4}}{\partial I_{v}}\right|=0<\infty
\end{array}\right\}
$$

For $f_{5}$, we have:

$$
\left.\begin{array}{l}
\left|\frac{\partial f_{5}}{\partial S_{c}}\right|=\left|\frac{\partial f_{5}}{\partial G}\right|=\left|\frac{\partial f_{5}}{\partial C}\right|=\left|\frac{\partial f_{5}}{\partial H}\right|=0<\infty \\
\left|\frac{\partial f_{5}}{\partial P_{v}}\right|=\left|-\left(\mu_{1}+\alpha\right)\right|<\infty \\
\left|\frac{\partial f_{5}}{\partial I_{v}}\right|=0<\infty
\end{array}\right\}
$$

And for $f_{6}$ :

$$
\left.\begin{array}{l}
\left|\frac{\partial f_{6}}{\partial S_{c}}\right|=\left|\frac{\partial f_{6}}{\partial G}\right|=\left|\frac{\partial f_{6}}{\partial C}\right|=\left|\frac{\partial f_{6}}{\partial H}\right|=\left|\frac{\partial f_{6}}{\partial P_{v}}\right|=|\alpha|<\infty \\
\left|\frac{\partial f_{6}}{\partial I_{v}}\right|=\left|-\mu_{1}\right|<\infty
\end{array}\right\}
$$

Since all the partial derivatives exist, continuous and are bounded, by Lemma 3.1, the model exists and the solution is unique. 


\section{Numerical Application}

Numerical solution of the propose model was investigated for better understanding. The system was solved using Differential Transform Method [1, 2]. The fundamental mathematical operations performed by differential transform method was adopted see [2] for more information.

Using the fundamental operators in [2], the following transform and recurrence relation of equation (1) were obtained

$$
\left.\begin{array}{rl}
S_{c}(k+1) & =\frac{1}{k+1}\left[\Lambda_{c}-\beta_{1} \sum_{m=0}^{k} I_{v}(m) S_{c}(k-m)-\mu S_{c}(k)\right] \\
G(k+1) & =\frac{1}{k+1}\left[\beta_{1} \sum_{m=0}^{k} I_{v}(m) S_{c}(k-m)-\gamma G(k)-\mu G(k)\right] \\
C(k+1) & =\frac{1}{k+1}[\gamma G(k)-(\mu+\epsilon) C(k)] \\
H(k+1) & =\frac{1}{k+1}[\epsilon C(k)-\mu H(k)] \\
P_{v}(k+1) & =\frac{1}{k+1}\left[\Lambda_{v}-\mu_{1} P_{v}(k)-\alpha P_{v}(k)\right] \\
I_{v}(k+1) & =\frac{1}{k+1}\left[\Lambda_{v}+\alpha P_{v}(k)-\mu_{1} I_{v}(k)\right]
\end{array}\right\}
$$

Equation (13) was solved with Maple 18 using data on Table 2.1 and the result obtained were shown graphically in Figures 2, 3 and 4.
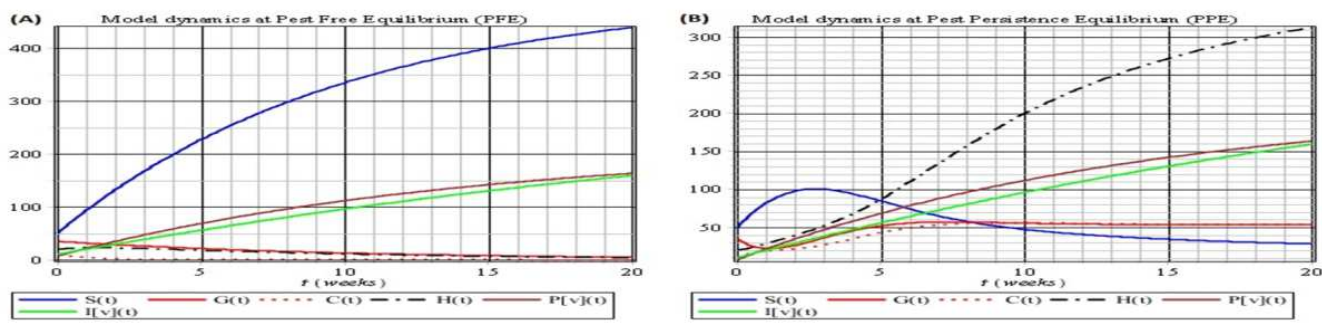

Figure 2: (A) The dynamics for which $I_{v}=0$, and (B) $I_{v} \neq 0$.
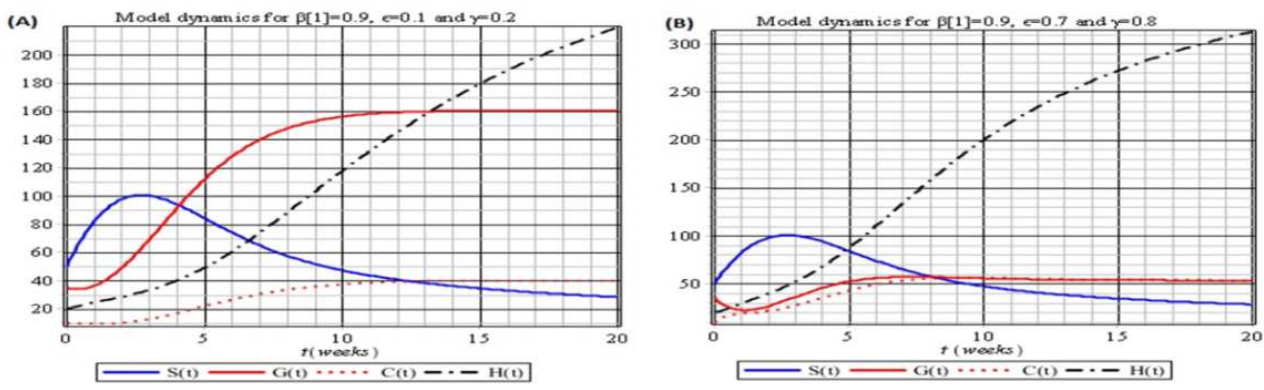

Figure 3: The dynamics for which $I_{v} \neq 0$. 

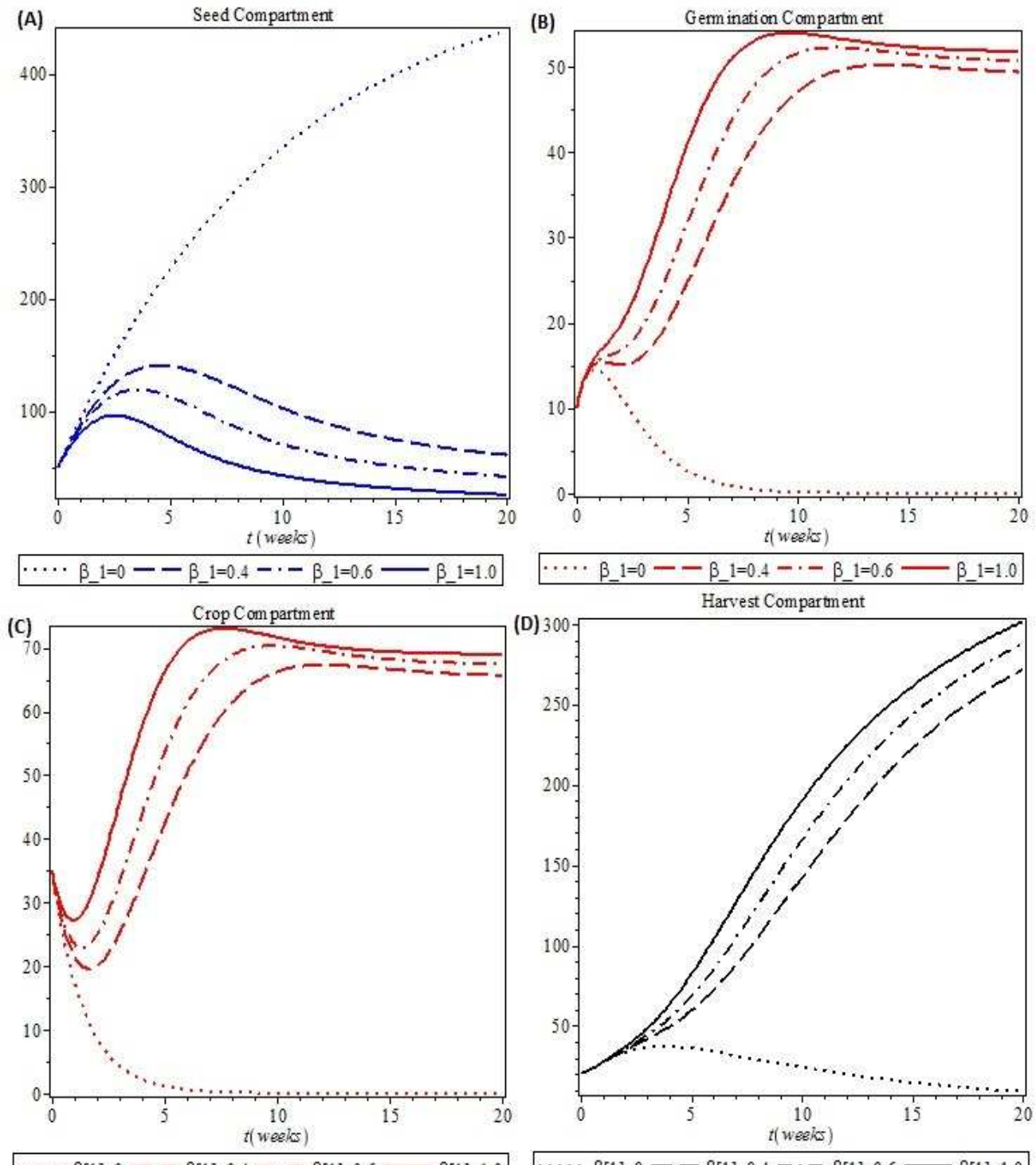

$\cdots \cdots \beta[1]=0-\beta[1]=0.4-\cdot-\beta[1]=0.6-\beta[1]=1.0$

$\cdots \cdot \beta[1]=0--\beta[1]=0.4-\cdot-\beta[1]=0.6-\beta[1]=1.0$

Figure 4: Variation of chances of attack on the sorghum (i.e. $\beta$ varying from $0, \ldots, 1$ ).

\section{Discussion of Results}

Figure 2(A) At Pest free Equilibrium, it was establish that, $S_{c}=\frac{\Lambda_{c}}{\mu}, G=0, C=$ $0, H=0$, which implies dynamics when there are no plantation and no pest attack the seed, only pest population and seed exist but there is no interaction between the pest and 
the seed since the seed are systemic and they are yet to be planted. While Figure 2(B) depicted the case at Pest Persistent Equilibrium, interaction of pest and sorghum seed after planting, during the germination process, pest was able to attack at about 3 weeks of planting which drastically reduce the germination process which also reduced the cropping process, though farmers were able to harvest but most of the harvest are bad.

From the assumption that $P_{v}$ helps the sorghum seeds to germinate and $I_{v}$ causes the damage on the plant. Figure 3(A) clearly explains the scenario which much germination was observed but less crops were produced and this affects the quantity of harvest. Figure 3(B) explains that right from the beginning of germination, the pest had a successful attack on the seed but the survival of the attack produce crop in proportion almost equal to the number of germination seeds. And this case show better harvest than when much germination and less crop. Figure 4 shows variation of chances of attack on the sorghum (i.e., $\beta$ varying from $0, \ldots, 1$ ).

\section{Conclusion}

The sorghum production model was formulated, characterized and analyzed with differential transform method. The result obtained shows that pest attacking the production of sorghum can be controlled at every stage with the introduction of beneficial insect so as to maximize the sorghum production. More so the climatic condition of the soil and states of seeds before planting are essential factor that determines the production of sorghum.

\section{References}

[1] F. S. Akinboro, S. Alao and S. O. Akinpelu, Numerical solution of SIR model using DTM and VIM, Journal of General Mathematics Notes 22(2) (2014), 82-92.

[2] K. C. Amit, S. Pabel and M. I. Anowarul, Analysis of epidemic model by differential transform method, Journal of Multidisciplinary Engineering Science and Technology (JMEST) (2017).

[3] T. N. Chuc, S. Vijaya, J. V. Erik, C. C. Scott, R. J. David and L. H. Graeme, Genetic variability in high temperature effects on seed-set in sorghum, Functional Plant Biology 40 (2013), 439-488. https://doi.org/10.1071/FP12264

[4] B. Debashis and P. Samares, Stability analysis of a non linear HIV/AIDS model with vaccination and antirotional therapy, International Journal of Advances in Applied Mathematics and Mechanics 5(2) (2017), 41-50. 
[5] S. A. Egbetade and M. O. Ibrahim, Modelling the Impact of BCG vaccines on tuberculosis epidemics, Journal of Mathematical Modelling and Application 9 (2014), 49-55.

[6] J. Howard Frank and Russell F. Mizell, common name: ladybirds, lady beetles, ladybugs [of Florida]; scientific name: (Insecta: Coleoptera: Coccinellidae), UF/IFAS Featured Creatures, 2018. http://entnemdept.ufl.edu/creatures/beneficial/lady_beetles.htm

[7] FAO, Food and Agricultural Organisation Statistic, 2012. Available at www.fao.org/faostat

[8] FAO, Food and Agricultural commodities production, FAOSTAT, Food and Agricultural Organization of the United Nations, 2013. http://faostat.fao.org/site /339/default.aspx

[9] P. C. Joseph, J. Dirk and S. Stefan, Modelling and analysis of integrated pest control strategies via impulsive differential equations, International Journal of Differential Equations 2017 (2017), Article ID 1820607, 18 pages.

https://doi.org/10.1155/2017/1820607

[10] Ric Bessin, Ladybugs, UK Cooperative Extension Services, University of KentuckyCollege of Agriculture, 2018. https://entomology.ca.uky.edu/ef105

[11] D. B. Robert, J. B. Michael, L. K. David, G. John, S. Nick, E. Norman, G. B. David, M. O. Way, T. A. Royer, B. Stephen and M. Enn, Sugarcane aphid (Hemiptera: Aphididae): A new pest on sorghum North America, Journal of Integrated Pest Management 7(1) (2016), 1-13. https://doi.org/10.1093/jipm/pmw011

[12] M. Sisterson and D. Stenger, Roguing with replacement in perennial crops: modelling conditions for successful disease management, Phytophathology 103 (2013), 117-128. https://doi.org/10.1094/PHYTO-05-12-0101-R

[13] F. Soltani, H. Khemira, S. Atifi and Q. Ghazwani, Mathematical characterization of growth of a local landrace of sorghum from Saudi Arabia, Int. J. Pure App. BioSci. 6(1) (2018), 52-55. https://doi.org/10.18782/2320-7051.6075

[14] USDA, Nigeria grain and feed annual, Annual report 2014, United States Department of Agriculture, GAIN Report, 2014.

[15] USDA, Nigeria grain and feed annual, Annual report 2017, United States Department of Agriculture, GAIN Report, 2017.

[16] S. B. Vahid, Stability analysis of positive systems with applications to epidemiological model, A dissertation for the degree of Doctor of Philosophy, National University of Ireland, Maynooth, 2012. 
[17] P. V. Vara and A. S. Scott, Soils, plant growth and crop production: growth and production of sorghum and millet, Encyclopedia of Life Support System (EOLSS) 2 (2010), 1-27.

This is an open access article distributed under the terms of the Creative Commons Attribution License (http://creativecommons.org/licenses/by/4.0/), which permits unrestricted, use, distribution and reproduction in any medium, or format for any purpose, even commercially provided the work is properly cited. 